\title{
Voice Analysis in Individuals with Chronic Obstructive Pulmonary Disease
}

\author{
${ }^{1}$ Anuradha Shastry, ${ }^{2}$ Radish Kumar Balasubramanium, ${ }^{3}$ Preetam R Acharya
}

\begin{abstract}
Background: Chronic obstructive pulmonary disease (COPD) is a respiratory condition that can affect voice as respiration is the source for voice production. There are very few studies available on the analysis of voice in individuals with COPD (asthma, chronic bronchitis and emphysema).
\end{abstract}

Purpose of the study: The aim of this study was to analyze the acoustic and perceptual characteristics of voice in individuals with COPD.

Method: The study followed a comparative cross-sectional study design. Sixty-four participants were considered, and they were divided into two groups (group 1: individuals with COPD, $n=32$; group 2: normal individuals, $n=32$ ). Acoustic analysis was performed using CSL software. The perceptual analysis was done using CAPE-V.

Results: The results showed that there was significant difference between the two groups on acoustic measures (lower fundamental frequency, reduced frequency range, increased pitch and amplitude perturbation measures and increased noise measures), and perceptual measures (presence of increased roughness, breathiness, strain).

Conclusion: We can conclude that COPD has an impact on voice measures as assessed through acoustic and perceptual parameters. These voice deviations may be due to COPD itself or due to the effects of the medication.

Clinical implication: The findings of this study provide valuable information regarding voice problems in the individuals with COPD so that an appropriate intervention plan can be designed.

Keywords: Chronic obstructive pulmonary disease, Chronic asthma, COPD, Acoustic measures, Perceptual measures, Voice analysis.

How to cite this article: Shastry A, Balasubramanium RK, Acharya PR. Voice Analysis in Individuals with Chronic Obstructive Pulmonary Disease. Int J Phonosurg Laryngol 2014;4(2):45-49.

Source of support: Nil

Conflict of interest: None

\footnotetext{
${ }^{1}$ Assistant Professor, ${ }^{2,3}$ Associate Professor

1,2Department of Audiology and Speech Language Pathology Kasturba Medical College, Manipal University, Mangalore Karnataka, India

${ }^{3}$ Department of Pulmonary Medicine, Kasturba Medical College Manipal University, Mangalore, Karnataka, India

Corresponding Author: Radish Kumar Balasubramanium Associate Professor, Department of Audiology and Speech Language Pathology, Kasturba Medical College, Manipal University, Mangalore, Karnataka, India, Phone: 08244286432 e-mail: radheesh_b@yahoo.co.in
}

\section{INTRODUCTION}

The global initiative for chronic obstructive lung disease (GOLD) defines chronic obstructive pulmonary disease (COPD) as 'A disease characterized by limitation of airflow that is not completely reversible. The airflow limitation is usually both progressive and is associated with an abnormal inflammatory response of the lungs to noxious particles or gases.' This kind of inflammation stimulates the production of excessive mucous (sputum), which causes further obstruction in the airway.

Chronic obstructive pulmonary disease has various causes; one of the leading causes is smoking/consumption of tobacco. Further, environmental factors and genetic influences can also heighten a person's likelihood of acquiring COPD. Long-term exposure to lung irritants that damage the lungs and the airway as a result of air pollution, occupational dusts, second-hand smoke and chemicals are some of the environmental factors. Few might acquire COPD due to Heredity factors which include a history of childhood respiratory infections/ COPD, while few others might develop COPD due to low levels of alpha- 1 antitrypsin (AAT) which is also known as the 'Lung Protector' is a protein made in the liver. COPD is a broad term that covers several lung conditions which include chronic bronchitis, emphysema and chronic asthma.

Common symptoms of COPD include chronic cough, dyspnea, xerostomia, frequent respiratory infections and dysphagia in severe cases. Apart from these symptoms, COPD can affect voice production and quality, directly because it is associated with respiratory decline, and indirectly due to the associated symptoms and the side effects of medication. Although, there is a scarcity of research on the effect of COPD on voice problems, some studies have focused on the impact of asthma on the voice measures.

Voice problems in these individuals have been neglected and increased prominence is given to assessment and treatment of the respiratory problem, despite the fact that the respiratory system is the source for voice production. These respiratory conditions are known to cause adverse effects on voice which might further affect the quality of life of the individual. Although, there is a scarcity of research on the voice problems in individuals with COPD, there are few studies that have been focused 
on the impact of asthma on the voice measures. ${ }^{2-5} \mathrm{How}-$ ever, there are no investigations carries out till date on the detailed analysis of voice in individuals with the other types of COPD. Hence, there is a need for a detailed analysis of voice in COPD patients so that a proper understanding about their voice problems can be obtained. In this regard, we assessed the voice characteristics in these individuals with chronic bronchitis, emphysema and Asthma using perceptual and acoustic measures.

\section{AIM OF THE STUDY}

The study aimed as assessing the acoustic and perceptual measures in individual with chronic obstructive pulmonary disease.

\section{METHODS}

\section{Participants}

Sixty-four participants (30 females and 34 males) were considered for this present study in two groups within the age range of 25 to 70 years with the mean age of 47.5 years. Group 1 consisted of individuals with COPD $(n=32)$ and group 2 consisted of age and gender matched controls $(n=32)$. All the individuals in group 1 were diagnosed with COPD by an experienced physician in the field of pulmonary medicine based on the signs, symptoms and lung function tests as per the GOLD standards. Individuals with mild to moderate degree of COPD were included in this group (Mild COPD: FEV1/FVC $<70 \%$, FEV1 $\geq 80 \%$ of predicted; moderate COPD: FEV1/FVC $<$ $70 \%, 50 \%$ of predicted $\leq$ FEV1 $<80 \%$ of predicted). The exclusionary criteria for both groups included individuals with the history of vocal abuse/misuse, surgery to the laryngeal structures, hearing impairment, neurological problems affecting the voice production and professional voice users.

\section{Instrumentation}

Acoustic analysis was performed using advanced multidimensional voice program (MDVP) and voice range profile (VRP) module using CSL software (Computerized Speech Lab) (Model 4150, Kay Elemetrics Corp). The consensus auditory-perceptual evaluation of voice (CAPE-V) is the perceptual voice rating scale used to describe the severity of auditory-perceptual attributes of voice problems. $^{6}$

\section{Procedure}

Research ethical approval was received from Manipal University's Institutional human research ethics committee, and the testing began only after obtaining the written consent from the participants.
Participants were seated comfortably on a chair. A microphone was used to record the speech sample in a sound-treated room for the purpose of perceptual and acoustic analysis. The mouth to microphone distance was kept at around $15 \mathrm{~cm}$.

\section{Acoustic Analysis}

The participants were asked to produce a sustained phonation of vowel /a/ for as long as possible at a comfortable pitch and loudness. Phonation of vowel /a/ was recorded in three trials using the same equipment. Acoustic analysis was done using MDVP advanced module and VRP module of the CSL software which analyzed the sample based on certain calculations and gives us robust information about the frequency related measures, perturbation measures, and noise related measurements.

\section{Perceptual Analysis}

Running speech sample (on the topic home) was recorded using the sound recorder. Three experienced speech pathologists perceptually rated the voice samples based on CAPE-V.

\section{Data Analysis}

The acoustic measures (fundamental frequency, frequency range, intensity range, Jitter, smoothed pitch pertubation quotient, shimmer, smoothed amplitude pertupation quotient, Noise harmonic ratio and soft phonation index) were robustly obtained through the CSL Software using the MDVP and SRP modules. Perceptual measures (overall severity, roughness, breathiness, strain, pitch, loudness and other parameters) were assessed using the CAPE-V rating scale.

\section{Statistical Analysis}

The values of all the measures were tabulated in the Microsoft excel sheet. The descriptive statistics were calculated for all measures. The perceptual ratings of the clinicians were subjected to Cronbach's Alpha analysis to determine the reliability of voice ratings. The scores obtained from the perceptual analysis were compared across the two groups using independent-samples t-test. The acoustic measures were averaged for both groups and two-way ANOVA test was performed to compare the measures across the groups.

\section{RESULTS}

The present study was carried out to investigate the effect of chronic obstructive pulmonary disease on voice production as respiratory system is crucial for voice production. Acoustic and perceptual measures were employed for the purpose. 


\section{Acoustic Measures}

Subsequent to tabulation of the data, the mean and the standard deviation for all the parameters were obtained for both the groups and the genders (Table 1).

From Table 1, it is apparent that the values differed across the two groups (COPD and normal controls). Two way ANOVA was the test performed to compare the acoustic measures across the groups and genders. Results revealed an overall significant difference between the COPD and normal group $(\mathrm{p}<0.05)$.

The COPD individuals had a significantly lower fundamental frequency $\mathrm{F}(1,60)=6.80, \mathrm{p}<0.001$, reduced frequency range at $\mathrm{F}(1,60)=8.21, \mathrm{p}<0.001$, higher pitch perturbation measures consisting of Jitter at $F(1,60)$ $=17.31$ and $\mathrm{p}<0.001$, sPPQat $\mathrm{F}(1,60)=33.9, \mathrm{p}<0.001$, higher amplitude perturbation measures consisting of shimmer at $\mathrm{F}(1,60)=18.27, \mathrm{p}<0.001$ and $\mathrm{sAPQ}$ at $\mathrm{F}$ $(1,60)=12.95, \mathrm{p}<0.001$, increased NHR at $\mathrm{F}(1,60)=6.55$, $\mathrm{p}=0.31$ when compared with the normal group. Although the intensity range did not show significant difference across the groups the raw mean scores were lower for the COPD group when compared to the normal group at $\mathrm{F}(1,60)=0.19, \mathrm{p}=0.662$. But, the raw mean SPI scores did not show significant difference across the groups $\mathrm{F}(1,60)=0.28, \mathrm{p}=0.596$.

The two groups showed a significant difference across the gender only with respect to fundamental frequency with females having a higher fundamental frequency when compared to males $\mathrm{F}(1,60)=117.09, \mathrm{p}<0.001$.
Table 2: Descriptive statistics for perceptual measures for both the groups

\begin{tabular}{lll}
\hline \multirow{2}{*}{$\begin{array}{l}\text { Perceptual } \\
\text { parameters }\end{array}$} & \multicolumn{2}{c}{ Mean and standard deviation } \\
\cline { 2 - 3 } & COPD & Normal controls \\
\hline Overall severity & $17.1563 \pm 9.13083$ & $1.2813 \pm 1.49569$ \\
Roughness & $16.8125 \pm 9.51002$ & $0.5938 \pm 1.25633$ \\
Breathiness & $12.1250 \pm 8.02674$ & $0.8438 \pm 1.33593$ \\
Strain & $9.9688 \pm 8.96726$ & $0.2813 \pm 0.98349$ \\
Pitch & $8.2188 \pm 5.87021$ & $0.1563 \pm 0.62281$ \\
Loudness & $9.9688 \pm 9.02020$ & $0.1406 \pm 0.66350$ \\
\hline
\end{tabular}

COPD: Chronic obstructive pulmonary disease

\section{Perceptual Measures}

The descriptive statistics were calculated for all the perceptual parameters (Table 2).

The ratings of both the clinicians were subjected to chronbach's alpha analysis to assess the reliability of the perceptual ratings. The results revealed a good agreement between both raters with the chronbach's alpha $\alpha=0.92$.

The raw mean scores presented for all the perceptual parameters differed across the groups with the COPD group having higher scores indicating increased vocal deviations in comparison to the normal populations. The results of independent-samples t-test revealed statistically significant difference across both the groups at $p<0.05$.

Independent-samples t-test indicated significantly higher scores in the COPD group than for normal controls for overall severity at $\mathrm{t}(126)=13.72, \mathrm{p}<0.001$, roughness at $\mathrm{t}(126)=13.52, \mathrm{p}<0.001$, breathiness at $\mathrm{t}(126)=11.09$, $\mathrm{p}<0.001$, strain at $\mathrm{t}(126)=8.59, \mathrm{p}<0.001$, pitch at $\mathrm{t}(126)$ $=10.92$ and loudness at $\mathrm{t}(126)=8.69, \mathrm{p}<0.001$.

Table 1: Descriptive statistics for acoustic measures for both the groups

\begin{tabular}{llll}
\hline \multirow{2}{*}{$\begin{array}{l}\text { Acoustic } \\
\text { parameters }\end{array}$} & Gender & COPD & Normal controls \\
\cline { 3 - 4 } Fundamental & Female & $191.0587 \pm 48.10788$ & $230.2877 \pm 22.09220$ \\
Frequency & Male & $131.1747 \pm 23.90662$ & $130.4441 \pm 15.22350$ \\
Frequency & Female & $80.3913 \pm 36.37893$ & $157.7993 \pm 101.67139$ \\
range & Male & $98.7341 \pm 108.68303$ & $142.5647 \pm 68.80467$ \\
Intensity range & Female & $23.0667 \pm 11.68312$ & $19.6000 \pm 9.27978$ \\
& Male & $18.6471 \pm 9.82307$ & $19.9412 \pm 8.56935$ \\
Jitter & Female & $2.5660 \pm 1.03733$ & $1.7656 \pm 0.88547$ \\
& Male & $2.5446 \pm 1.59207$ & $1.0025 \pm 0.75646$ \\
Shimmer & Female & $4.9300 \pm 3.56812$ & $2.6774 \pm 1.23583$ \\
& Male & $5.8023 \pm 3.04433$ & $2.7052 \pm 1.28528$ \\
sPPQ & Female & $2.3525 \pm 1.02705$ & $1.0847 \pm 0.49227$ \\
& Male & $3.0540 \pm 1.97603$ & $0.8016 \pm 0.50659$ \\
sAPQ & Female & $7.4035 \pm 5.79775$ & $3.3445 \pm 0.89703$ \\
& Male & $8.5246 \pm 6.93989$ & $4.2279 \pm 1.49592$ \\
SPI & Female & $26.5145 \pm 11.27354$ & $34.6491 \pm 40.25633$ \\
& Male & $28.9264 \pm 24.56347$ & $27.4000 \pm 12.99123$ \\
NHR & Female & $0.1585 \pm 0.06085$ & $0.1379 \pm 0.05648$ \\
& Male & $0.1641 \pm 0.06943$ & $0.1113 \pm 0.03740$ \\
\hline COPD: Chronic obstructive pulmonary disease; NHR: Noise to harmonic ratio; \\
sAPQ: Smoothened amplitude perturbation quotient; SPI: Soft phonation index; \\
sPPQ: Smoothened pitch perturbation quotient &
\end{tabular}


In addition to these parameters reduced breath support was observed in $46.87 \%$ of 32 individuals with COPD, vocal fry was observed in $6.25 \%$ of 32 COPD individuals and diplophonia was present in $3.12 \%$ of 32 individuals with COPD.

\section{DISCUSSION}

The present study investigated the acoustic aerodynamic and perceptual voice measures in individuals with COPD and normal controls. The results indicated that there was significant difference between both the groups for the acoustic, aerodynamic and perceptual voice parameters indicating that the individuals with COPD had more deviated vocal parameters in comparison to normal controls.

\section{Acoustic Analysis}

The results of the acoustic analysis showed an overall statistically significant difference between the COPD group and normal group. This means that the voices of both the groups differed in the acoustic parameters.

The COPD group had lower fundamental frequency values attributing to the decreased respiratory support leading to reduced subglottal pressure in them. Consequently, this might have led to the increased mass and decreased tension of the vocal folds resulting in the lower fundamental frequency values in them. However, these results are contradicting the results of Asnaashari et al ${ }^{4}$ who reported no significant difference in fundamental frequency parameter between the asthmatics and controls. However, this difference in the findings could be due to the fact that only asthmatics were considered in their study.

Similarly, the COPD group had a significantly lower frequency range compared to normal controls suggestive of the inability of the vocal folds to modulate the frequency during a speech task unlike the normal controls. Further, due to the presence of an abnormal vibratory pattern of the vocal folds, the frequency range might have been reduced subsequently.

Statistical analysis revealed no significant difference for the intensity range between the COPD and the normal group. However, clinically significant difference was observed. The lack of significant difference might be due to the large variability among the COPD subjects as shown by the larger standard deviation for both genders.

The results of pitch perturbation measures (Jitter and sPPQ) showed that the COPD group had higher pitch perturbation compared to the normal group which suggests instability in the vocal fold vibratory pattern during phonation and increased aperiodicity which is in line with the findings by Williamson et al and Dogan, et al.
Findings from the statistical test disclosed that COPD group had larger amplitude perturbation measures (Shimmer and SAPQ) compared to the normal group indicative of the inability of the individuals with COPD to maintain the intensity during phonation. This could also be attributed to a large variation in the intensity being perpetuated at the level of the vocal folds. This is in agreement with the previous study by Williamson, et al and Dogan et al. ${ }^{2,3}$

The values obtained for NHR were shown to be significantly higher in the COPD group which could be due to increased breathiness in the voice due to reduced sub glottal pressure built up below the vocal folds of the individuals with COPD because of which presence of noise in the spectrum was higher in them. However, SPI did not significantly differ across the two groups which indicates that there was no certain probability of any mass related vocal fold pathology in these individuals. On the other hand, the SPI values were found to be extensively variable within each group which could be ascribed to the point that a small percentage of normal population also demonstrate an incomplete glottal closure/possess a glottis chink.

Statistical analysis for gender comparison showed that fundamental frequency values were significantly different across the genders with females having higher values compared to males which was expected due to the characteristics of the vocal tract configuration that is gender specific. Further as witnessed from the individual values as well as the results from the statistical analysis it appears that the gender did not show any effect on the frequency range, intensity range, pitch perturbation measures, amplitude perturbation measures as well as for noise measures.

\section{Perceptual Measures}

The results of statistical analysis also showed a significant difference between the two groups for all perceptual measures. The COPD group had significantly higher Roughness component (mild to moderate deviancy), higher breathiness (mild to moderate deviancy), higher strain (milddeviancy), deviated pitch (mild deviancy) and deviated loudness (mild deviancy). This could be due to the irregularity in the vocal fold vibration due to the obstructive air flow limitation, thereby leading to incoordination between the subglottal pressure and vocal fold vibratory pattern. The overall severity rating was also higher in the COPD group indicative of the global, integrated impression of voice deviance which is attributed to the point that the limited respiratory support due to COPD causes the alteration in the laryngeal functioning leading to increased roughness, breathiness, harshness 
along with pitch and loudness deviations in the voice. All these findings correlated well with the findings of the acoustic analysis.

In addition to these parameters, $46.87 \%$ of the individuals in the COPD group had reduced breath support, $6.25 \%$ of the individuals in the COPD had vocal fry, and $3.12 \%$ of individuals with COPD had diplophonia dring the phonation and speaking tasks. This finding is in line with the fact that individuals with COPD have a respiratory compromise (reduced lung volumes and capacities) due to which they are unable to sustain the phonation for speech. Also, these individuals have decreased respiratory support leading to insufficient subglottal pressure to facilitate vocal fold contact. This in turn results in the bubbling of air leading to the popping kind of sound leading to vocal fry. All these parameters suggest that COPD has an impact on the voice quality of the individuals manifesting the symptoms.

\section{CONCLUSION}

The present study investigated the voice characteristics in individuals with COPD using acoustic measures and perceptual measures. The results revealed that there was a significant difference between the two groups on all the measures indicating the presence of vocal deviations in individuals with COPD. Further, studies should address the vocal fold vibratory characteristics and the efficacy of vocal treatment measures in these individuals with COPD.

\section{ACKNOWLEDGMENTS}

The authors would like to thank Manipal University for funding this study.

\section{REFERENCES}

1. Pauwels RA, Buist AS, Calverley PM, et al. Global strategy for the diagnosis, management, and prevention of chronic obstructive pulmonary disease. NHLBI/WHO Global Initiative for Chronic Obstructive Lung Disease (GOLD) Workshop summary. Am J Respir Crit Care Med 2001;163(5): 1256-1276.

2. Williamson IJ, Matusiewicz SP, Brown PH, Greening AP, Crompton GK. Frequency of voice problems and cough in patients using pressurized aerosol inhaled steroid preparations. Eur Respir J 1995;8(4):590-592.

3. Dogan M, Eryuksel E, Kocak I, Celikel T, Sehito M. Subjective and objective evaluation of voice quality in patients with asthma. J Voice 2007;21(2):224-230.

4. Asnaashari AM, Rezaei S, Babaeian M, Taiarani M, Shakeri MT, Fatemi SS, Darban AA. The effect of asthma on phonation: a controlled study of 34 patients. Ear Nose Throat J 2012;91(4):168-171. Available at: http://www.thefreelibrary.com/ The+effect+of+asthma+on+ phonation $\% A+a+$ controlled+stud y+of+34+patients.-a028887 2086

5. Cassiani RA, Aguiar-Ricz L, Santos CM, Martinez JAB, Dantas. Glottal competence in chronic obstructive pulmonary disease. Audiology Communication Research 2013;18(3): 149-154. Available at: http://www.scielo.br/pdf/acr/v18n3/ en_a03v18n3.pdf

6. Kempster GB, Gerratt BR, Verdolini-Abbott K, BarkmeierKraemer J, Hillman RE. Consensus auditory-perceptual evaluation of voice: development of a standardized clinical protocol. Am J Speech Lang Pathol 2009;18(2):124-132. 